\title{
A genetic analysis of Trichuris trichiura and Trichuris suis from Ecuador
}

\author{
Hayley Meekums ${ }^{1}$, Mohamed BF Hawash ${ }^{2,3}$, Alexandra M Sparks ${ }^{4,5}$, Yisela Oviedo ${ }^{6}$, Carlos Sandoval ${ }^{6}$, \\ Martha E Chico ${ }^{6}$, J Russell Stothard ${ }^{4}$, Philip J Cooper ${ }^{6,7,8}$, Peter Nejsum ${ }^{2}$ and Martha Betson ${ }^{1 *}$
}

\begin{abstract}
Background: Since the nematodes Trichuris trichiura and T. suis are morphologically indistinguishable, genetic analysis is required to assess epidemiological cross-over between people and pigs. This study aimed to clarify the transmission biology of trichuriasis in Ecuador.

Findings: Adult Trichuris worms were collected during a parasitological survey of 132 people and 46 pigs in Esmeraldas Province, Ecuador. Morphometric analysis of 49 pig worms and 64 human worms revealed significant variation. In discriminant analysis morphometric characteristics correctly classified male worms according to host species. In PCR-RFLP analysis of the ribosomal Internal Transcribed Spacer (ITS-2) and 18S DNA (59 pig worms and 82 human worms), nearly all Trichuris exhibited expected restriction patterns. However, two pig-derived worms showed a "heterozygous-type" ITS-2 pattern, with one also having a "heterozygous-type" 185 pattern. Phylogenetic analysis of the mitochondrial large ribosomal subunit partitioned worms by host species. Notably, some Ecuadorian T. suis clustered with porcine Trichuris from USA and Denmark and some with Chinese T. suis.
\end{abstract}

Conclusion: This is the first study in Latin America to genetically analyse Trichuris parasites. Although T. trichiura does not appear to be zoonotic in Ecuador, there is evidence of genetic exchange between T. trichiura and T. suis warranting more detailed genetic sampling.

Keywords: Trichuris, Whipworm, Human, Pig, Ecuador, Zoonosis, Phylogenetics

\section{Findings}

\section{Background}

The soil-transmitted helminth (STH) Trichuris trichiura infects around 465 million people worldwide [1], being especially prevalent where hygiene and sanitation are poor. The closely-related $T$. suis infects innumerable pigs globally and is associated with significant economic losses [2]. As eggs, larvae and adults of T. trichiura and T. suis are morphologically indistinguishable, the extent of natural cross-transmission of Trichuris between humans and pigs is not known. Experimental infection studies reveal that $T$. trichiura can establish in pigs, although adult worms rarely persist, while patent $T$. suis infection has been observed in man [3].

The introduction of molecular techniques has provided insights into genetic differences between T. trichiura and

\footnotetext{
* Correspondence: mbetson@rvc.ac.uk

'Department of Production and Population Health, Royal Veterinary College, Hawkshead Lane, Hatfield, Herts AL9 7TA, UK

Full list of author information is available at the end of the article
}

T. suis and the potential for cross-transmission between host species. Analysis of mitochondrial and nuclear sequences in pig-derived and human-derived Trichuris has supported the proposition that T. trichiura and $T$. suis are distinct species $[4,5]$. However, transmission from pigs to humans in Uganda has been noted [6]. The transmission biology of trichuriasis in other parts of the world, e.g. Latin America, awaits investigation. In Ecuador, the estimated prevalence of T. trichiura infection is 9\% [7], but prevalences are much higher in certain rural areas $[8,9]$. Epidemiological surveillance of T. suis in pigs is scant, but given that there are over 1.2 million pigs in the country [10] and backyard production systems are common, $T$. suis infections are likely to be widespread.

The aims of this study were to perform a genetic analysis of Trichuris collected from pigs and people in rural Ecuador to clarify the epidemiology of trichuriasis. 
Table 1 Morphometric characteristics of male Trichuris derived from humans and pigs in Ecuador

\begin{tabular}{|c|c|c|c|c|c|}
\hline \multirow[t]{2}{*}{ Characteristic } & \multicolumn{2}{|c|}{ Human-derived Trichuris $(\mathrm{n}=28)$} & \multicolumn{2}{|c|}{ Pig-derived Trichuris $(n=6)$} & \multirow[t]{2}{*}{ P-value } \\
\hline & Median (IQR) ${ }^{a}$ & Min.-Max. & Median (IQR) & Min.-Max. & \\
\hline Anterior length & $20(18-21)$ & $4-27$ & $25.5(23.5-27.75)$ & $19-30$ & 0.006 \\
\hline Posterior length & $11(10.25-13)$ & $6-17$ & $15(13.75-16)$ & $13-16$ & 0.002 \\
\hline Total length & $32(28.25-34.75)$ & $15-40$ & $41.5(38-42.5)$ & $32-44$ & 0.002 \\
\hline Anterior width & $0.08(0.07-0.10)$ & $0.06-0.17$ & $0.12(0.08-0.15)$ & $0.07-0.16$ & 0.06 \\
\hline Posterior width & $0.48(0.43-0.54)$ & $0.37-0.82$ & $0.69(0.55-0.99)$ & $0.55-1.00$ & 0.0006 \\
\hline Spicule length & $2.66(2.56-2.73)$ & $1.51-3.01$ & $2.44(2.14-2.89)$ & $2.11-2.96$ & 0.21 \\
\hline
\end{tabular}

All measurements are in $\mathrm{mm} .^{\mathrm{a}}$ Interquartile range.

\section{Methods}

A parasitological survey of humans and pigs was conducted in Quinidé and Súa Districts, Esmeraldas Province, Ecuador [11]. 32 families $(n=132)$ were enrolled. Pigs $(n=46)$ were selected for sampling based on their proximity to Trichuris-positive children. Participants were asked to provide a faecal sample and faecal samples were collected from pigs. Parasitological diagnosis was conducted by examination of duplicate Kato-Katz smears [12]. Ethical approval was provided by the Ethical Committees of Liverpool School of Tropical Medicine and Pontificia Universidad Catolica del Ecuador [11]. Written informed consent was provided by all participants or their guardians (for children). STH-positive individuals were treated with $400 \mathrm{mg}$ albendazole or pyrantel pamoate (see below).

To collect adult worms, participants with $T$. trichiura counts $\geq 480$ eggs per gram faeces were given pyrantel pamoate at $10 \mathrm{mg} / \mathrm{kg}$ over three days. Participants and their guardians were instructed to collect stools produced. Recovered worms were washed thoroughly before storage in 70\% ethanol. Trichuris-positive pigs were treated with piperazine at $0.2 \mathrm{~g} / \mathrm{kg}$. Expelled worms were collected over the following two days. Morphometric analysis of adult Trichuris was carried out as described [6]. Discriminant analysis was conducted in SPSSv20 starting with four morphometric characteristics (female worms), which were removed or exchanged in a stepwise fashion to identify the most discriminatory combination. A maximum of three characteristics was used for male worms due to the small number obtained from pigs. The data for female worms did not meet the assumption of equal variance among groups.

Genomic DNA was extracted from Trichuris using the Wizard Genomic DNA Purification Kit. Internal Transcribed Spacer-2 (ITS-2) PCR-RFLP was conducted as described [6]. An 18S DNA PCR-RFLP was designed. Restriction sites were identified using Webcutter2.0 and Alu1 differentiated between human and pig Trichuris. The primers were: Tri18S_F1 (5'- CGAACGAGACTC TGGCCTAC) and Tri18S_R (5' - CCTTGTTACGACT TTTACTTCCTC). Cycling conditions were: denaturation at $95^{\circ} \mathrm{C}$ for $15 \mathrm{~min}$, followed by 35 cycles of $95^{\circ} \mathrm{C}$ for $30 \mathrm{~s}, 55^{\circ} \mathrm{C}$ for $1 \mathrm{~min}$ and $72^{\circ} \mathrm{C}$ for $1 \mathrm{~min}$, with a final extension of $72^{\circ} \mathrm{C}$ for $5 \mathrm{~min}$. PCR products $(4 \mu \mathrm{l})$ were digested with $4 \mathrm{U}$ Alu1.

29 worms (15 from four humans and 14 from a pen containing four pigs) were chosen for mitochondrial large ribosomal subunit $(r r n \mathrm{~L})$ sequencing. $422 \mathrm{bp}$ of $r r n \mathrm{~L}$ were amplified using primers TrirrnLF $\left(5^{\prime}\right.$-TGTA AWTCTCCTGCCCAATGA) and TrirrnLR (5'-CGGTT TAAACTCAAATCACGTA). PCR conditions were: initial denaturation at $95^{\circ} \mathrm{C}$ for $15 \mathrm{~min}$ followed by 35 cycles of $95^{\circ} \mathrm{C}$ for $30 \mathrm{~s}, 50^{\circ} \mathrm{C}$ for $30 \mathrm{~s}$ and $72^{\circ} \mathrm{C}$ for $1 \mathrm{~min}$ and a final extension at $72^{\circ} \mathrm{C}$ for $10 \mathrm{~min}$. For comparison, 10 pig worms from Denmark and USA were analysed and additional sequences were retrieved from GenBank: Chinese T. suis (AM993027-AM993032, HQ183734HQ183736 and GU070737) and T. trichiura (AM993 017-AM993022). Phylogenetic analysis using neighborjoining and maximum-likelihood trees was conducted in MEGA6.1 [13] using jModelTest to identify the best

Table 2 Morphometric characteristics of female Trichuris derived from humans and pigs in Ecuador

\begin{tabular}{|c|c|c|c|c|c|}
\hline \multirow[t]{2}{*}{ Characteristic } & \multicolumn{2}{|c|}{ Human-derived Trichuris $(n=36)$} & \multicolumn{2}{|c|}{ Pig-derived Trichuris $(n=43)$} & \multirow[t]{2}{*}{ P-value } \\
\hline & Median (IQR) $^{a}$ & Min.-Max. & Median (IQR) & Min.-Max. & \\
\hline Anterior length & $20.5(14.5-25)$ & $5-35$ & $25(18-32)$ & $2-38$ & 0.03 \\
\hline Posterior length & $10(9-12)$ & $5-17$ & $10(8-12)$ & $3-22$ & 0.28 \\
\hline Total length & $30(24-36.75)$ & $14-47$ & $33(28-44)$ & $11-55$ & 0.12 \\
\hline Anterior width & $0.09(0.07-0.10)$ & $0.06-0.18$ & $0.10(0.08-0.12)$ & $0.05-0.17$ & 0.18 \\
\hline Posterior width & $0.57(0.54-0.62)$ & $0.49-0.83$ & $0.69(0.52-0.79)$ & $0.34-1.05$ & 0.02 \\
\hline
\end{tabular}

All measurements are in $\mathrm{mm}^{\mathrm{a}}{ }^{\mathrm{a}}$ Interquartile range. 


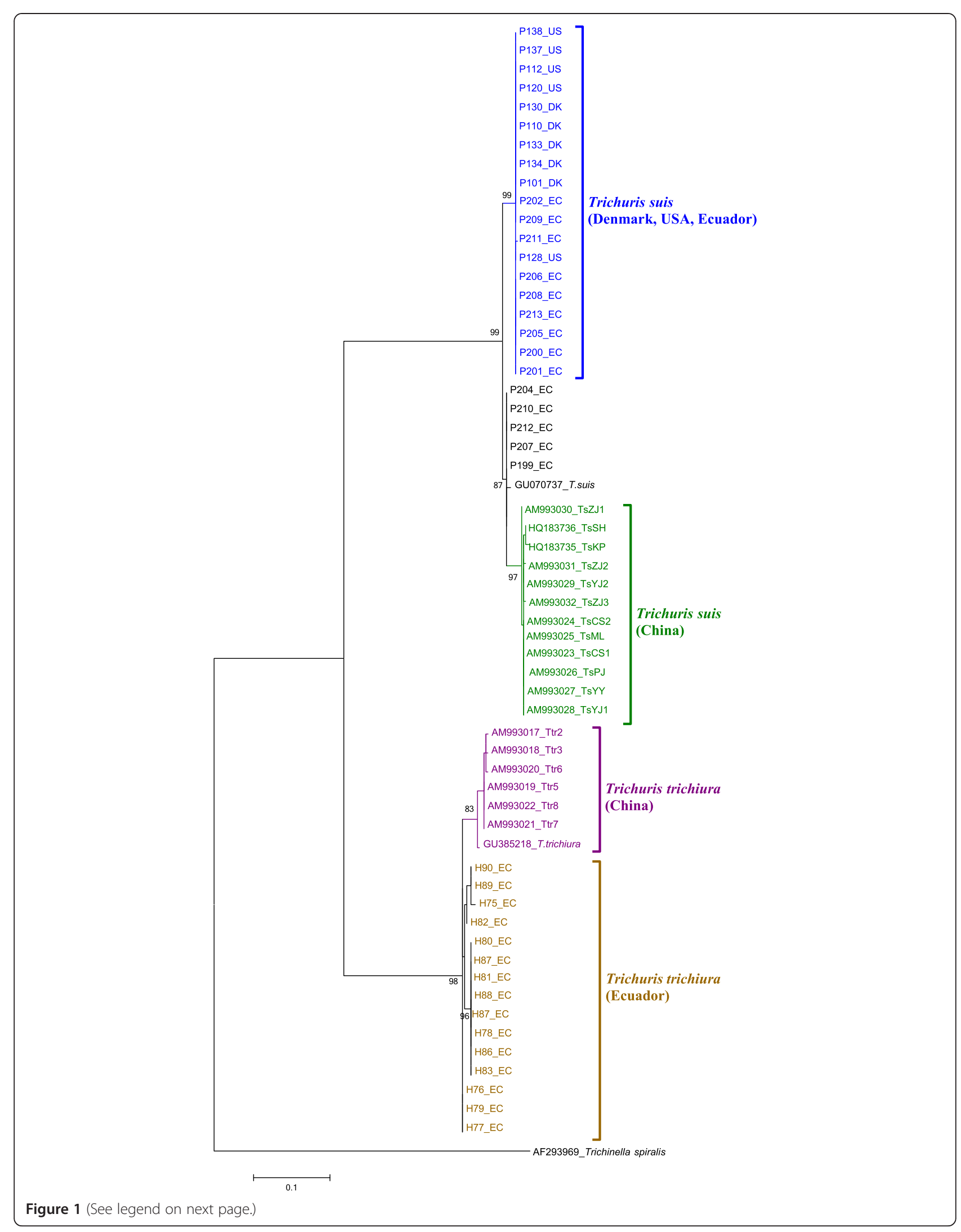


(See figure on previous page.)

Figure 1 Maximum likelihood tree based on the rrnL gene using Tamura-Nei with gamma distribution as the substitution model and Trichinella spiralis as an outgroup. Bootstrap values above $\mathbf{8 0}$ are reported. Scale bar: number of base substitutions per site. Sample key: first letter indicates host ( $\mathrm{H}$ - human, P- pig); last two letters indicate country of origin (UG - Uganda, EC - Ecuador, DK - Denmark, US - USA);

numerals indicate unique worm ID. A neighbour joining tree showed a very similar topography.

model [14]. Trichinella spiralis (AF293969) was used as an outgroup. Ecuadorian $r r n \mathrm{~L}$ sequences were submitted to GenBank (KP781884-KP781912).

\section{Results}

The prevalence of trichuriasis among human participants was $46.2 \%$ (95\%CI: $37.5-55.1 \%$ ) and of 46 pigs sampled, five were egg-positive. After chemoexpulsion 697 Trichuris were collected from 10 humans, and 62 Trichuris were obtained from a pen containing four pigs.

Morphometric analysis was conducted on 49 pig worms and 64 human worms (Tables 1 and 2). There was a tendency for pig worms to be longer than human worms, with differences in total, anterior and posterior body lengths between male pig and human worms. Spicule length did not differ between male worms from pigs and humans. Discriminant analysis using posterior length, anterior length and posterior width correctly classified 100\% of male worms $(n=29)$ with respect to host species, but only $69.3 \%$ of female worms $(n=75)$.

PCR-RFLP analysis was carried out on 59 pig worms (one pig pen) and 82 human worms (four hosts). All human-derived worms showed identical ITS-2 restriction patterns (bands at 340, 220 and $130 \mathrm{bp}$ ) and $18 \mathrm{~S}$ patterns (360 and $170 \mathrm{bp}$ ). The majority of pig-derived worms demonstrated the same restriction pattern for ITS2 (490 and $130 \mathrm{bp}$ ) and $18 \mathrm{~S}$ bands at 360, 130 and $40 \mathrm{bp}$. Two pig Trichuris showed a "heterozygous-type" ITS-2 pattern (490, 340, 220 and $130 \mathrm{bp}$ ). One of these also showed a "heterozygous-type" $18 \mathrm{~S}$ pattern. It was not possible to resolve the $18 \mathrm{~S}$ pattern for the other sample.

Upon phylogenetic analysis of the mitochondrial $r r n \mathrm{~L}$ marker, all Ecuadorian human-derived worms clustered in a distinct group but in the same clade as Chinese human-derived worms (Figure 1). Ecuadorian pigderived worms were separated into two groups with affiliations to pig worms from Denmark and USA or to Chinese T. suis. The $r r n \mathrm{~L}$ sequences for pig Trichuris which showed heterozygous-type ITS-2 and 18S patterns (P207_EC and P208_EC) clustered with Ecuadorian pig Trichuris sequences.

\section{Discussion}

Here we present the first comparison of Trichuris worms derived from pigs and people in Latin America, specifically in rural Ecuador. We observed significant differences in certain morphometric characteristics, but there was morphological overlap between worms from different host species. We found that pig worms were longer than human worms in Ecuador, whereas human worms were longer in Uganda [3]. Consistent with the literature [15], discriminant analysis was able to correctly classify male worms, but not female worms, with respect to host origin. Given the fact that they can represent adaptations to a particular host species [16], morphometric characteristics must be interpreted with caution when used to distinguish between Trichuris species.

In the two PCR-RFLP analyses, all human-derived worms showed restriction patterns characteristic of worms derived from humans in other locations (ITS-2) [6] or that were expected based on in silico analyses of GenBank sequences (18S). Similarly, all mitochondrial rrnL gene sequences from human worms clustered together in a distinct clade from the Ecuadorian pigderived worms. Thus, there was no evidence of zoonotic transmission of Trichuris worms from pigs to people, in contrast to Uganda [6].

Intriguingly, two out of 59 pig-derived worms showed "heterozygous-type" restriction patterns in the ITS-2 and $18 \mathrm{~S}$ (in one case) PCR-RFLPs, the first time heterozygotes or mixed template profiles have been noted in pig Trichuris. This pattern may be explained by cross-infection and genetic exchange between Trichuris species by introgression or hybridization or through retention of ancestral polymorphisms within multi-copy ribosomal arrays that predated speciation [17]. As the Trichuris prevalence in humans is higher than in pigs, perhaps due to rapid expulsion of the worm population in pigs [18] or regular pig deworming (A.S., O.M.Pogoreltseva, personal communication), in this setting it may be more likely for pigs to become infected with human Trichuris than vice versa. Nevertheless, a more extensive analysis of Trichuris from pig and human hosts is necessary to confirm this and place the genetic exchange event within a likely time-frame.

The mitochondrial $r r n \mathrm{~L}$ gene analysis confirmed the genetic distinction between human and pig Trichuris [4]. Ecuadorian and Chinese human worms were found in two groups showing phylo-geographic isolation. Some Ecuadorian pig Trichuris clustered with T. suis from Denmark and the USA, and some with Chinese T. suis. Pigs were originally imported into Ecuador from the Iberian Peninsula during Spanish colonization, with subsequent imports from Europe and the USA over the course of the 20th century [19]. Thus genetic similarity between T. suis from Ecuador and T. suis from Europe and USA is 
not surprising. The similarity of five worms to T. suis from China, maybe due to the introduction of European and Chinese pig breeds in the 20th century when pigs were imported from China to enhance commercial traits of European breeds [20].

\section{Conclusions}

Molecular epidemiological studies can provide important insights into parasite transmission dynamics. Although there appears to be no zoonotic transmission of Trichuris in Ecuador, there is evidence of (ancestral) genetic exchange between $T$. trichiura and $T$. suis, warranting additional genetic sampling.

\section{Competing interests}

The authors declare that they have no competing interests.

\section{Authors' contributions}

$\mathrm{HM}$ carried out the morphometric analysis. $\mathrm{HM}, \mathrm{MBF}$ and $\mathrm{MB}$ conducted the molecular genetic studies and MBF carried out the phylogenetic analysis. AS, YO, CS and MEC conducted the field survey and collected the Trichuris. PJC, JRS, MB and PN designed the study. MB drafted the manuscript with assistance from PN. All authors read and approved the final manuscript.

\section{Acknowledgments}

$\mathrm{HM}$ and MB acknowledge the financial support of the Royal Veterinary College, UK. AS and JRS received financial support from Liverpool School of Tropical Medicine, UK. Sample collection in Ecuador was supported by a Wellcome Trust grant 088862/Z/09/Z to PJC. Analysis of worms was supported by a Danish Agency for Science, Technology and Innovation grant awarded to PN.

\section{Author details \\ 'Department of Production and Population Health, Royal Veterinary College, Hawkshead Lane, Hatfield, Herts AL9 7TA, UK. ²Department of Veterinary Disease Biology, Faculty of Health and Medical Sciences, University of Copenhagen, Dyrlaegevej 100, Frederiksberg C DK-1870, Denmark. ${ }^{3}$ Zoology Department, Faculty of Science, Cairo University, Giza 12613, Egypt. ${ }^{4}$ Department of Parasitology, Liverpool School of Tropical Medicine, Pembroke Place, Liverpool L3 5QA, UK. Institute of Immunology and Infection Research, Centre for Immunity, Infection and Evolution, School of Biological Sciences, University of Edinburgh, King's Buildings, Ashworth Laboratories, Charlotte Auerbach Road, Edinburgh EH9 3FL, UK. 'Laboratorio de Investigaciones FEPIS, Quinindé, Esmeraldas Province, Ecuador. ${ }^{7}$ Centro de Investigaciónen Enfermedades Infecciosas, Pontificia Universidad Católica del Ecuador, Quito, Ecuador. ${ }^{8}$ Institute of Infection and Immunity, St George's University of London, Cranmer Terrace, London SW17 ORE, UK.}

Received: 14 January 2015 Accepted: 3 March 2015

Published online: 19 March 2015

\section{References}

1. Pullan RL, Smith JL, Jasrasaria R, Brooker SJ. Global numbers of infection and disease burden of soil transmitted helminth infections in 2010. Parasit Vectors. 2014;7:37.

2. Roepstorff A, Mejer H, Nejsum P, Thamsborg SM. Helminth parasites in pigs: new challenges in pig production and current research highlights. Vet Parasitol. 2011;180:72-81

3. Nejsum P, Betson M, Bendall RP, Thamsborg SM, Stothard JR. Assessing the zoonotic potential of Ascaris suum and Trichuris suis: looking to the future from an analysis of the past. J Helminthol. 2012;86:148-55.

4. Liu GH, Gasser RB, Su A, Nejsum P, Peng L, Lin RQ, et al. Clear genetic distinctiveness between human- and pig-derived Trichuris based on analyses of mitochondrial datasets. PLoS Negl Trop Dis. 2012;6:e1539.

5. Callejon R, Nadler S, De Rojas M, Zurita A, Petrasova J, Cutillas C. Molecular characterization and phylogeny of whipworm nematodes inferred from
DNA sequences of cox1 mtDNA and 185 rDNA. Parasitol Res. 2013;112:3933-49.

6. Nissen S, Al-Jubury A, Hansen TV, Olsen A, Christensen H, Thamsborg SM, et al. Genetic analysis of Trichuris suis and Trichuris trichiura recovered from humans and pigs in a sympatric setting in Uganda. Vet Parasitol. 2012:188:68-77.

7. Chammartin F, Scholte RG, Guimaraes LH, Tanner M, Utzinger J, Vounatsou P. Soil-transmitted helminth infection in South America: a systematic review and geostatistical meta-analysis. Lancet Infect Dis. 2013;13:507-18.

8. Cooper PJ, Chico ME, Bland M, Griffin GE, Nutman TB. Allergic symptoms, atopy, and geohelminth infections in a rural area of Ecuador. Am J Respir Crit Care Med. 2003;168:313-7.

9. Moncayo AL, Vaca M, Amorim L, Rodriguez A, Erazo S, Oviedo G, et al. Impact of long-term treatment with ivermectin on the prevalence and intensity of soil-transmitted helminth infections. PLoS Negl Trop Dis. 2008;2:e293.

10. FAOSTAT [http://faostat3.fao.org/faostat-gateway/go/to/browse/Q/QA/E]

11. Sparks AM, Betson M, Oviedo G, Sandoval C, Cooper PJ, Stothard JR. Characterization of Ascaris from Ecuador and Zanzibar. J Helminthol. 2014; doi:dx.doi.org/10.1017/S0022149X14000431

12. Katz N, Chaves A, Pellegrino J. A simple device for quantitative stool thick-smear technique in Schistosomiasis mansoni. Rev Inst Med Trop Sao Paulo. 1972;14:397-400.

13. Tamura K, Stecher G, Peterson D, Filipski A, Kumar S. MEGA6: Molecular Evolutionary Genetics Analysis version 6.0. Mol Biol Evol. 2013;30:2725-9.

14. Posada D. jModelTest: phylogenetic model averaging. Mol Biol Evol. 2008;25:1253-6.

15. Spakulova M. Discriminant-analysis as a method for the numerical evaluation of taxonomic characters in male trichurid mematodes. Syst Parasitol. 1994;29:113-9.

16. Knight RA. Morphological differences in Trichuris ovis associated with different host species. J Parasitol. 1984;70:842-3.

17. Anderson TJ. The dangers of using single locus markers in parasite epidemiology: Ascaris as a case study. Trends Parasitol. 2001;17:183-8.

18. Nejsum P, Thamsborg SM, Petersen HH, Kringel H, Fredholm M, Roepstorff A. Population dynamics of Trichuris suis in trickle-infected pigs. Parasitology. 2009;136:691-7.

19. Ortiz B. Los cerdos criollos ecuatorianos. In: Los Cerdos Locales en los Sistemas Tradicionales de Producción. Roma: FAO; 2001. p. 37-70.

20. Bosse M, Megens HJ, Frantz LA, Madsen O, Larson G, Paudel Y, et al. Genomic analysis reveals selection for Asian genes in European pigs following human-mediated introgression. Nat Commun. 2014;5:4392.

\section{Submit your next manuscript to BioMed Central and take full advantage of:}

- Convenient online submission

- Thorough peer review

- No space constraints or color figure charges

- Immediate publication on acceptance

- Inclusion in PubMed, CAS, Scopus and Google Scholar

- Research which is freely available for redistribution 